\title{
Abstract
}

\section{Wearable XAI: A Knowledge-Based Federated Learning Framework ${ }^{\dagger}$}

\author{
Sara Nasiri ${ }^{1,2, *}$, Iman Nasiri ${ }^{2}$ and Kristof Van Laerhoven ${ }^{1}(\mathbb{D}$ \\ 1 Department of Electrical Engineering and Computer Science, University of Siegen, 57076 Siegen, Germany; \\ kvl@eti.uni-siegen.de \\ 2 SmartyX GmbH (Node 4.0), Martinshardt. 19, 57074 Siegen, Germany; iman@smartyx.ai \\ * Correspondence: sara.nasiri@uni-siegen.de \\ + Presented at the 8th International Symposium on Sensor Science, 17-28 May 2021; Available online: \\ https://i3s2021dresden.sciforum.net/.
}

Citation: Nasiri, S.; Nasiri, I.; Van Laerhoven, K. Wearable xAI: A Knowledge-Based Federated Learning Framework. Eng. Proc. 2021, 6, 79. https://doi.org/10.3390/ I3S2021Dresden-10143

Academic Editors: Gianaurelio Cuniberti and Larysa Baraban

Published: 17 May 2021

Publisher's Note: MDPI stays neutral with regard to jurisdictional claims in published maps and institutional affiliations.

Copyright: (C) 2021 by the authors. Licensee MDPI, Basel, Switzerland. This article is an open access article distributed under the terms and conditions of the Creative Commons Attribution (CC BY) license (https:// creativecommons.org/licenses/by/ $4.0 /)$.

\begin{abstract}
Federated learning is a knowledge transmission and training process that occurs in turn between user models on edge devices and the training model in the central server. Due to privacy policies and concerns and heterogeneous data, this is a widespread requirement in federated learning applications. In this work, we use knowledge-based methods, and in particular case-based reasoning (CBR), to develop a wearable, explainable artificial intelligence (xAI) framework. CBR is a problem-solving AI approach for knowledge representation and manipulation, which considers successful solutions of past conditions that are likely to serve as candidate solutions for a requested problem. It enables federated learning when each user owns not only his/her private data, but also uniquely designed cases. New generated cases can be compared to the knowledge base and the recommendations enable the user to communicate better with the whole system. It improves users' task performance and increases user acceptability when they need explanations to understand why and how AI algorithms arrive at these optimal solutions.
\end{abstract}

Keywords: artificial intelligence; wearable AI; mobile edge computing; case-based reasoning; recommender system

Supplementary Materials: The presentation file is available at https:/ / www.mdpi.com/article/10.3 390/I3S2021Dresden-10143/s1.

Institutional Review Board Statement: Not applicable.

Informed Consent Statement: Not applicable. 